\title{
Influence of cysteine 164 on active site structure in rat cysteine dioxygenase
}

DOI:

10.1007/s00775-016-1360-0

Document Version

Accepted author manuscript

Link to publication record in Manchester Research Explorer

\section{Citation for published version (APA):}

Fellner, M., Siakkou, E., Faponle, A., Tchesnokov, E. P., De Visser, S., Wilbanks, S. M., \& Jameson, G. N. L. (2016). Influence of cysteine 164 on active site structure in rat cysteine dioxygenase. Journal of Biological Inorganic Chemistry, 21(4), 501-510. https://doi.org/10.1007/s00775-016-1360-0

\section{Published in:}

Journal of Biological Inorganic Chemistry

\section{Citing this paper}

Please note that where the full-text provided on Manchester Research Explorer is the Author Accepted Manuscript or Proof version this may differ from the final Published version. If citing, it is advised that you check and use the publisher's definitive version.

\section{General rights}

Copyright and moral rights for the publications made accessible in the Research Explorer are retained by the authors and/or other copyright owners and it is a condition of accessing publications that users recognise and abide by the legal requirements associated with these rights.

\section{Takedown policy}

If you believe that this document breaches copyright please refer to the University of Manchester's Takedown Procedures [http://man.ac.uk/04Y6Bo] or contact uml.scholarlycommunications@manchester.ac.uk providing relevant details, so we can investigate your claim.

\section{OPEN ACCESS}




\section{Influence of Cysteine 164 on Active Site Structure in Rat Cysteine Dioxygenase}

Matthias Fellner, $†$ Eleni Siakkou,$\dagger$ Abayomi S. Faponle,, Egor P. Tchesnokov,$\dagger$ Sam P. de Visser,I Sigurd M. Wilbanks,

$\dagger$ Department of Chemistry and $\ddagger$ Department of Biochemistry, University of Otago, PO Box 56, Dunedin 9054, New Zealand. IManchester Institute of Biotechnology and School of Chemical Engineering and Analytical Science, The University of Manchester, 131 Princess Street, Manchester M1 7DN, United Kingdom

$\square$ To whom correspondence should be addressed: Department of Chemistry, University of Otago, PO Box 56, Dunedin 9054 fax: +64 3479 7906, tel: +64 3479 8028, email: guy.jameson@otago.ac.nz

Abbreviations: CDO, cysteine dioxygenase; CSA, cysteine sulfinic acid. 


\begin{abstract}
Cysteine dioxygenase (CDO) is a non-heme mononuclear iron enzyme with unique structural features, namely an intramolecular thioether crosslink between cysteine 93 and tyrosine 157, and a disulfide between substrate L-cysteine and cysteine 164 in the entrance channel to the active site. We investigated how these posttranslational modifications affect catalysis through a kinetic, crystallographic and computational study. The enzyme kinetics of a C164S variant are identical to WT indicating that disulfide formation at C164 does not significantly impair access to the active site at physiological $\mathrm{pH}$. However, at high $\mathrm{pH}$ the cysteine-tyrosine crosslink formation is enhanced in $\mathrm{C} 164 \mathrm{~S}$. This supports the view that disulfide formation at position 164 can limit access to the active site. The $\mathrm{C} 164 \mathrm{~S}$ yielded crystal structures of unusual clarity in both resting state and with cysteine bound. Both show that the iron in the cysteine bound complex is a mixture of penta- and hexa-coordinate with a water molecule taking up the final site $(60 \%$ occupancy), which is where dioxygen is believed to coordinate during turnover. The serine also displays stronger hydrogen bond interactions to a water bound to the amine of the substrate cysteine. However, the interactions between cysteine and iron appear unchanged. DFT calculations support this and show that WT and C164S have similar binding energies for the water molecule in the final site. This variant therefore provides evidence that WT also exists in this equilibrium between penta- and hexa-coordinate forms and presence of the sixth ligand does not strongly affect dioxygen binding.
\end{abstract}

Key Words: Cysteine dioxygenase, post-translational modification, ES coordination, kinetics, DFT. 


\section{Introduction}

The mononuclear non-heme iron enzyme cysteine dioxygenase (CDO, EC 1.13.11.20) converts L-cysteine and molecular oxygen to cysteine sulfinic acid. CDO is part of a larger class of enzymes, the thiol dioxygenases, that catalyze formation of the corresponding sulfinate from a range of different thiols. This group includes cysteamine dioxygenase, [1] 3-mercaptopropionate dioxygenase[2-5] and mercaptosuccinate dioxygenase, [6] which have been found to have a range of substrate specificities. CDO catalyzes the first step in oxidative cysteine breakdown in mammals.[7] Tight regulation of cysteine levels has been shown to be important for health and linked to many diseases with recent studies also linking CDO activity to human cancer. [8,9]

The active site ferrous iron in mammalian CDO is chelated by a 3-histidine motif in close proximity to an unusual posttranslational modification, a cysteine-tyrosine crosslink. Although the cysteine and tyrosine residues that form the crosslink are completely conserved in eukaryotes, a glycine replaces the cysteine in most bacterial sequences where CDO activity remains. [10] Interestingly, other thiol dioxygenases appear to also lack the crosslink, demonstrating that the crosslink is not required for activity. Nonetheless, we and others have shown that mammalian CDO activity is dependent upon the amount of crosslink.[11, 12] Indeed, the kinetic parameters of the two isoforms were shown to be quite different.[13]

A second posttranslational modification forms in mammalian CDO; a disulfide between cysteine 164 and exogenous cysteine. This was initially observed crystallographically[14] and subsequently characterized by us using mass spectrometry.[15] Indeed, the first cysteine bound structure for CDO (PDB ID 2ICI) [16] has been reanalyzed and interpreted as containing such a disulfide.[17] Furthermore, sequence alignment and structural comparison shows that although cysteine is conserved at this position in CDO of multicellular eukaryotes, it is replaced by a 
catalytically important arginine residue in 3-mercaptopropionate dioxygenases.[3] This disulfide is believed to be important because cysteine 164 lies at the entrance to the channel to the active site.

However, removal of cysteine 164 from CDO has been reported to lead to reduction in activity and not an increase as one might expect if only steric factors are considered. This reduction in activity has been shown in both human[16] and rat $\mathrm{CDO},[18]$ but the size of the decrease reported is quite different, depending on substrate concentration. To resolve seemingly conflicting statements about the role of cysteine 164, we re-investigated the C164S variant to explore what changes in activity could be observed and to understand if these could be explained structurally.

\section{Materials and Methods}

Expression, purification and kinetic assays of the WT and C164S CDO variant. Anaerobic expression and purification of C164S CDO was performed as described previously.[12] Aspurified protein was enriched with ferrous iron as and excess iron removed by Chelex, already described in greater detail elsewhere. $[12,13,19]$ The crosslink fraction was $\sim 50 \%$ as previously demonstrated for WT. [12] Activity assays were performed by HPLC-ELSD [20] at $37^{\circ} \mathrm{C}$ in phosphate buffer between $\mathrm{pH} 5.7$ and 7.5.

All cysteine solutions, including dilutions from a stock, were freshly prepared in buffer at the beginning of each experiment and adjusted to the desired $\mathrm{pH}$. The final protein concentration in all assays was 1.5-3.0 $\mu \mathrm{M}$. CDO activity and crosslink formation was typically assessed over a period up to 3 hours. The initial linear proportion of the product formation curves was used to analyse Michaelis-Menten type kinetics. All kinetic parameters were calculated relative to active iron containing protein. The fraction of crosslinked CDO in relation to non-crosslinked CDO was 
determined densitometrically from SDS-PAGE gel images using the program ImageQuant ${ }^{\mathrm{TM}} \mathrm{TL}$ (GE Healthcare, UK). Data were analyzed using Microsoft Excel 2007/2010 and GraphPad Prism version 5.00 for Windows.

Crystallization and Structural Determination of the resting state and cysteine bound C164S $C D O$ variant. Crystals were grown and processed using the same conditions described previously. [13, 21] Hanging drops of $2 \mu \mathrm{L}$ of approximately $50 \mathrm{mg} / \mathrm{mL}$ C164S CDO (10 mM sodium phosphate and $20 \mathrm{mM} \mathrm{NaCl}, \mathrm{pH} 7.5$ ) that had been reconstituted with iron and $1.5 \mu \mathrm{L}$ of reservoir buffer containing crushed WT CDO seeds were allowed to equilibrate above the reservoir buffer $(26 \%(\mathrm{w} / \mathrm{v})$ polyethylene glycol 4000, $200 \mathrm{mM}$ ammonium acetate, and $100 \mathrm{mM}$ sodium citrate, $\mathrm{pH}$ 6.3). Crystals grew within two days as thick needles up to $1 \mathrm{~mm}$ in length. For the resting state, data were collected at the home source at the University of Otago Department of Biochemistry at a rotating anode (Rigaku MSC 007HF copper anode with Osmic VariMax optics) using an image plate detector (Raxis IV++). With the crystal at $93 \mathrm{~K}$ and X-rays of wavelength of $1.542 \AA, 300$ images were collected at an exposure time of two minutes, using $0.5^{\circ}$ oscillation and a detector distance of $100 \mathrm{~mm}$. For the cysteine bound structure, a crystal was soaked for six minutes in a drop containing the reservoir solution mentioned above and 100 $\mathrm{mM}$ cysteine, with an adjusted $\mathrm{pH}$ of 8.1. This approach was based on a report by Driggers et al. [17] Data were collected in a similar manner to the resting state; 400 images were collected, using $0.5^{\circ}$ oscillation and a detector distance of $110 \mathrm{~mm}$. Data collection for both structures was done by CrystalClear-d*TREK. [22] Data were indexed in space group $\mathrm{P}_{3} 2_{1} 2$, with unit cell dimensions of $\sim 58,58$, and $122 \AA$ (Table 1), and integrated in iMosflm. [23] Scaling was done using Aimless, $[24,25] 5 \%$ of reflections were reserved for the calculation of $\mathrm{R}_{\text {free }}$ using the MTZ from $4 \mathrm{KWJ}$ as a reference. The resolution (Table 1 ) was cut back based $\mathrm{I} / \sigma \mathrm{I}>2$, which resulted 
in $\mathrm{CC} 1 / 2$ values of $>0.8$ (all values reported by Aimless). [24] PDB entry 4KWJ [21] with important sidechains substituted by alanines was used for molecular replacement in Phenix.Phaser [26] with one monomer in the symmetric unit. The active site iron atom was placed using Phaser-EP [26] (MR-SAD). Phenix.Refine, [26] Phenix.Readyset, [26] and COOT [27] were used for refinement. The cysteine model and constrains were created using Phenix.eLBOW.[28] MolProbity [29] was used to validate the structures. Coordinates and structure factors were deposited in the Protein Data Base, for the resting state as entry 4YYO, for the cysteine bound as entry $4 Z 82$.

Computational chemistry. All structures were optimized using the Gaussian-09 software package using models based on the crystal structure coordinates.[30] A minimum level of constraints was included in the model to enable fidelity in the structural representation of the active region. Our original model[31] consisted of the iron bound to the cysteine substrate with imidazole groups representing histidine residues 86, 88, 140 and 155. However, here we expanded the histidine groups to methylimidazole. As before, also included were the methylguanidinium group to represent arginine 60, a phenolate group in place of tyrosine 157 and methylsulfide for cysteine 93 . We further expanded the model with the methylsulfide group of cysteine 164, a bridging water molecule towards the carboxylate of the substrate and the phenolate group of tyrosine 58 . We calculated the resting state structure with a water molecule bound to the sixth ligand position for WT as well as the C164S mutant. These systems have an overall charge of +1 and were calculated in the quintet spin state only.

Geometry optimisations were carried out for each structure using the unrestricted hybrid density functional method B3LYP, $[32,33]$ with a basis set containing $6-31 \mathrm{G}+$ double- $\zeta$ basis set on all atoms except for the iron where a double- $\zeta$ quality LACVP basis set that includes an 
effective core potential was used (basis set BS1).[34] Additional calculations were done with the triple- $\zeta$ quality LACV3P+ basis set on iron (with core potential) and $6-311+\mathrm{G}^{*}$ on the rest of the atoms (basis set BS2). Vibrational frequencies were calculated on the optimised structures using the Gaussian-09 software package and free energies were calculated at $298.15 \mathrm{~K}$ and 1 atm, which resulted in local minima with real frequencies only.

\section{Results}

C164S CDO activity is the same as WT. The activities of C164S and WT were compared in phosphate buffer at $\mathrm{pH} 5.7,6.5$ and 7.5 at $37^{\circ} \mathrm{C}$ (Figure 1). For both, activity increases with increasing $\mathrm{pH}$ as observed previously for WT.[19] Kinetic parameters are indistinguishable at $\mathrm{pH}$ $5.7\left(k_{\text {cat }}=0.04 \pm 0.07 \mathrm{~s}^{-1}\right.$ and $\left.K_{\mathrm{m}}=3 \pm 2 \mathrm{mM}\right)$ and pH $6.5\left(k_{\mathrm{cat}}=0.40 \pm 0.07 \mathrm{~s}^{-1}\right.$ and $K_{\mathrm{m}}=11 \pm 2$ $\mathrm{mM})$. At $\mathrm{pH} 7.5 k_{\text {cat }}$ is not significantly different $\left(\mathrm{C} 164 \mathrm{~S} k_{\text {cat }}=0.48 \pm 0.07 \mathrm{~s}^{-1}\right.$ and $K_{\mathrm{m}}=5 \pm 2$ $\mathrm{mM}$; WT $k_{\text {cat }}=0.40 \pm 0.07 \mathrm{~s}^{-1}$ and $K_{\mathrm{m}}=9 \pm 2 \mathrm{mM}$ ). However, differences in $K_{\mathrm{m}}$ do suggest that C164S is more catalytically efficient than WT at this higher $\mathrm{pH}$.

Overall reaction profile and crosslink formation. Over longer time periods, cysteine depletion and CSA formation were seen to be sigmoidal (Figure 2). An initial linear phase (0-30 min) fulfilling the Michaelis-Menten requirements described above was observed. This was followed by a second faster phase ( $\sim 30-90 \mathrm{~min})$ and eventually a slower phase ( $90-180 \mathrm{~min})$ as substrate concentrations were depleted. The HPLC-ELSD method[20] allows both substrate depletion and product formation to be monitored and the sum of substrate consumed and product produced was constant throughout the time-course within the error of the method for each set of data, indicating that non-enzymatic oxidation of cysteine was insignificant during the experiment, suggesting a CSA:cysteine coupling of $\sim 100 \%$. 
During assays the overall crosslink formation as a function of time could be followed by SDSPAGE and densitometry. As described previously for WT CDO,[12] crosslink formation occurs almost simultaneously with CSA production (Figure 2). Crosslink formation was $\mathrm{pH}$ dependent with an almost linear increase in the final amount of crosslink formed with $\mathrm{pH}$ (Figure 2). Slightly more crosslink formation was observed using C164S than WT and nearly $100 \%$ crosslink was formed at high $\mathrm{pH}$, something we have not observed under our conditions with WT CDO.

Comparison of C164S resting state structure with WT. The crystal structure of C164S CDO variant shows a similar overall structure compared to WT CDO. The active site also appears to be largely unchanged with only subtle changes. The cysteine 93 tyrosine 157 crosslink is present and as in WT appears to be the only isoform that crystallizes. Analysis of the diffraction data was aided by anomalous scattering by sulfur atoms, e.g. cysteines 76, 93 and 130 and methionines 73 , 117 and 179 show significant anomalous peaks (Figure 3A). Although not optimized for exploitation of the anomalous signal from these atoms, the $\mathrm{Cu} K_{\alpha}$ radiation used for data collection gives both iron and sulfur have sufficiently large $f^{\prime \prime}$ ( 3.15 and 0.56 , respectively) to provide interpretable maps. In fact a strong anomalous signal was observed, most likely because of a combination of the wavelength $(1.542 \AA)$, the very large crystal $(\sim 1 \mathrm{~mm})$ and the relatively high overall resolution $(1.77 \AA)$. As expected no anomalous peak was observed for serine 164 .

Superposition with the WT CDO structure PDB 4KWJ[21] (Figure 3B) shows that the sidechain of residue 164 has almost the same orientation in both structures. The active site iron is again chelated by the 3 histidine residues ( 86,88 and 140), however a chloride molecule was modeled instead of bound water opposite histidine 86. A chloride molecule accounts better for the observed density, makes chemical sense and is certainly present in the crystallization 
conditions used. However, the B factor of the chloride molecule is significantly higher (44.1 $\left.\AA^{2}\right)$ compared to the average protein atom $\left(22.1 \AA^{2}\right)$ and the iron atom $\left(14.7 \AA^{2}\right)$, suggesting disorder. We therefore have to state that the modeling of chloride here is speculative and several disordered oxygen atoms could also be possible. We have observed a similar density close to the iron atom for other CDO structures and therefore conclude that the "chloride ion" is not directly related to the $\mathrm{C} 164 \mathrm{~S}$ substitution.

C164S CDO variant in complex with cysteine. A crystal soaked with $100 \mathrm{mM}$ cysteine at $\mathrm{pH} 8.1$ diffracted to produce data that clearly revealed a cysteine molecule bound to the active site iron. A strong anomalous signal for the iron and the sulfur of the bound cysteine confirms presence of cysteine and aids placement. A $m F_{o}-D F_{c}$ electron density map produced from a model with no iron, cysteine or water atoms and no sidechains for arginine 60, cysteine 93 and tyrosine 157 provides an unbiased view of the electron density in the active site (Figure 3C). The fully refined model and electron density are given in Figure 3D.

Overall the cysteine bound structures at $\mathrm{pH} 8.1$ of C164S and WT (4IEV [17]) are very similar with an RMSD of $0.18 \AA$ using a C $\alpha$ atom alignment (Figure 3E). [35] Driggers et al. [17] reported that some structural changes occurred at higher $\mathrm{pH}$ in the asparagine 76 loop and the same changes are observed with C164S. The only slight differences between the structures are seen in partial occupancy of additional alternate conformations in the C164S structure for residues asparagine 71, glutamic acid 127, histidine 173 (all outside the active site), and threonine 95 (within the vicinity of the active site). Likewise, comparison of the active site shows high similarities. Even residue 164 is very similar with the serine occupying the same rotamer as cysteine in the WT structure. Only methionine 179 occupies a different sidechain orientation in C164S compared to WT consistent with the disorder observed for this residue in 
other structures. This view is supported by the fact that no significant anomalous signal was observed for sulfur of residue 179 in comparison with, for example, methionine 73 which appears to be highly ordered (Figure 3D).

The most notable difference between the two structures is that in the C164S cysteine bound structure, unaccounted electron density next to the iron opposite histidine 86 remained after the cysteine ligand was modeled. A water molecule was placed at this position and its occupancy and B-factor were refined to $60 \%$ and $28.11 \AA^{2}$. The cysteine ligand molecule occupancy and Bfactor refinement resulted in $91 \%$ and $25.08 \AA^{2}$; while the iron atom was refined at $100 \%$ occupancy resulting in a B-factor of $21.06 \AA^{2}$ (Table 1).

Closer inspection shows that the hydrogen bonding between cysteine/serine 164 and the bound cysteine differs. The $\gamma$-sulfur/oxygen of cysteine/serine 164 is hydrogen bonded to a water molecule that is in turn hydrogen bonded to the amine of the substrate bound to the iron. The serine oxygen to water oxygen bond distance in $\mathrm{C} 164 \mathrm{~S}$, is shorter than the cysteine sulfur to water oxygen distance in WT and methionine 179 (although more disordered, see above) moves to take up the space (Figure 4). It is unclear whether the stronger serine-water interaction stabilizes the additional water molecule opposite histidine 86 . However, the hydrogen bonding between arginine 60 and the carboxylate of bound cysteine is unchanged.

Computational chemistry. To investigate the hydrogen bonding interactions between cysteine/serine and the bound substrate and water, computational studies were carried out. Optimized geometries are shown in Figure 5. The mutation of cysteine 164 to serine has a significant effect on the local charge distribution nearby the carboxylate group of the cysteine substrate. Thus, the oxygen atom of serine has a charge of -0.70 , whereas a sulfur atom in WT only has a charge of -0.10 . As a result, a serine moiety in position 164 will pull the bridging 
water molecule closer: $\mathrm{H}_{2} \mathrm{O}---\mathrm{HOSer}$ is $1.74 \AA$, whereas the corresponding bond in WT is $1.93 \AA$ (cf. $3.3 \AA$ O---O and 3.6 $\AA$ O---S distances in the corresponding crystal structures, respectively). This interaction also affects the bond strength between the bridging water molecule and the amide group of the substrate. In addition, the carboxylate group of the substrate and the crystal water molecule is considerably closer to the substrate in the mutant. Only very small changes in the metal ligand distances are seen.

The binding energy of the water to make the substrate bound complex hexa-coordinate was also calculated for both WT and C164S. The energies were found to be only $0.1 \mathrm{kcal} / \mathrm{mol}$ different suggesting that WT is also partially hexa-coordinate.

\section{Discussion}

Simmons et al. [36] and Ye et al. [16] independently of each other, noticed unexpected electron density distal to the $\mathrm{S} \gamma$-atom of residue $\mathrm{C} 164$ when analyzing crystal structure data from mammalian CDO crystals that had been either co-crystallised with or soaked in cysteine, but neither group was able to unambiguously identify the origin of this feature by refinement of their crystal structure models. Simmons and colleagues, however, found that this electron density was only observed in the structure of CDO in presence of substrate, but not in its absence. Thus it was proposed that the structural change at C164 was caused by intermolecular disulfide formation with cysteine. This theory has been strengthened recently by a reanalysis of the Ye structure by Driggers et al. [17]. Such reactions on cysteine residues are not uncommon in proteins.[37] It was further suggested that, in consequence, the disulfide would negatively affect CDO activity by obstructing access of substrate to the active site. Ye and co-workers put this hypothesis to the test by designing CDO variants where $\mathrm{C} 164$ was replaced by either serine or alanine, both amino acids incapable of forming a disulfide with substrate cysteine. Increased 
activity in those variants would have reinforced Simmons' theory, but the results showed an abatement of approximately $20 \%$ with respect to the activity of WT CDO instead. The origin of the unexpected electron density at C164 thus remained unclear. Kleffmann et al. [15] investigated covalent post-translational modifications in CDO by MALDI-TOF-MS and found that the modification at $\mathrm{C} 164$ after incubation with supraphysiological concentrations of substrate was indeed a mixed disulfide with cysteine.

The Michaelis Menten parameters of C164S and WT CDO that we present are comparable within error, suggesting that only at high cysteine concentrations and higher $\mathrm{pH}$ does the disulfide at position 164 interfere with reactivity or modification. Indirect evidence supports this proposal. The reaction profiles measured over longer time scales using $\mathrm{C} 164 \mathrm{~S}$ are more reproducible than WT but this is difficult to quantify. The final \% crosslink formed is, however, accurately quantified using SDS-PAGE densitometry. It is seen that $\mathrm{C} 164 \mathrm{~S}$ is more readily crosslinked than WT, especially at higher $\mathrm{pH}$ (Figure 2B). At higher $\mathrm{pH}$ the cysteine thiol is deprotonated and thus more reactive and more likely to form the disulfide. Once formed, the disulfide at position 164 impedes access to the active site and thus abrogates crosslink formation, which has been shown to require cysteine. $[15,18]$ Conversely, it could be the negative charge on the thiolate itself that might impede access. Either way, the C164S variant is unable to deprotonate in this $\mathrm{pH}$ range and/or form this disulfide and therefore is able to be fully crosslinked, in contrast to WT. In contrast to our results with WT, Pierce and coworkers have been able to form fully crosslinked WT mouse CDO,[11] something we have been unable to do using our protein and methods.

The C164S mutation appeared to have little significant effect on the overall structure. The resting state is nearly identical to the WT structure. In X-ray crystallographic models, the iron 
environment in resting states of CDO is variously modeled, with one [14], two [13, 17, 21] or three water molecules (when the metal was modeled as a nickel atom [38]) bound. For the resting state of the $\mathrm{C} 164 \mathrm{~S}$ variant we present a chloride ion as our best model to account for the observed electron density. In general it appears that disordered solvent molecules are exchanged at the iron atom and several interpretations of this disorder can be found in the different crystal structures of $\mathrm{CDO}$. The resting states of $\mathrm{CDO}$ structures also show different orientations of arginine 60 and methionine 179, with disulfide formation on residue cysteine 164 influencing their positions. For C164S, only one orientation of arginine 60 is observed in the resting state: pointing towards the active site and a corresponding methionine 179 position.

The orientation of cysteine bound to the C164S structure is unambiguous due to strong anomalous scattering by iron and various sulfur atoms, in particular of the ligand bound sulfur, providing strong independent support for the mode of L-cysteine binding described by Driggers et al.[17] While the cysteine is orientated very similarly to cysteine in the WT structure obtained under similar conditions, the C164S mutation does affect the hydrogen bonding network between residue 164 and the bound substrate. Importantly, there is also extra electron density next to the iron that can be modeled with a water molecule with $60 \%$ occupancy to make the iron hexacoordinate. This is intriguing because previous spectroscopic investigations of CDO have suggested that the cysteine bound complex is penta-coordinate, with the sixth coordination site free to allow dioxygen binding at this position. The exception to this is a recent study of the H155A variant of mouse CDO that showed through magnetic circular dichroism that the cysteine bound complex is 6 coordinate.[39] This difference in coordination number is explained by the alanine substitution breaking the hydrogen bonding network between residue 155 and tyrosine 157 that points towards the substrate coordination site (Figure 5). In our case, the mutation at 
position 164 also affects the hydrogen bonding network but from the opposite side of the active site and interacts with the substrate rather than the dioxygen binding site. We further probed its contribution through computational calculations.

The DFT calculations confirm the differences in the hydrogen bonding network and these can be explained by differences in the charge of the oxygen versus sulfur in serine and cysteine. However, no appreciable differences in the iron ligand environment was observed suggesting that, in contrast to the $\mathrm{H} 155 \mathrm{~A}$ variant, the substitution cannot explain the differences in coordination number. Indeed, the binding energies of water to the penta-coordinate cysteine bound structures of WT and C164S are almost indistinguishable. We therefore believe that the CDO enzyme substrate complex exists as a mixture of 5- and 6-coordinate, but this equilibrium is altered by the $\mathrm{H} 155 \mathrm{~A}$ variant.

The paradigm for dioxygen binding at non-heme mononuclear iron enzymes is currently based around data collected for enzymes with 2-histidine 1-carboxylate coordination and predominantly those that use $\alpha$-ketoglutarate as co-substrate.[40] This model involves initial substrate binding that causes the conversion of the iron center from 6- to 5-coordinate. The enzyme is now ready to coordinate dioxygen and it has been suggested that this allows the enzyme to control reactivity and only implement dioxygenation when sufficient substrate is available. However, a weakly coordinating water molecule occupying the sixth coordination site has been observed both crystallographically[41] and spectroscopically[42] in the halogenases SyrB2 and CytC3 respectively. In this case, it has been suggested that 6-coordination is important for ensuring halogenation rather than hydroxylation. It is therefore surprising that CDO allows a mixture of 5- and 6-coordination for the substrate bound complex prior to dioxygen binding. It could be that the difference lies in the neutral 3-histidine coordination, 
which allows substitution of water by dioxygen but further study is required to fully understand this process.

Our crystal structure of the cysteine bound $\mathrm{C} 164 \mathrm{~S}$ variant and related calculations improve our description of the ES complex in CDO. Altogether we describe the C164S variant and show that although it does affect cysteine-tyrosine crosslink formation, it does not appear to directly affect reactivity in the lower $\mathrm{pH}$ region. Cysteine 164 may act as a gate across the entrance channel, impeding access, particularly at higher $\mathrm{pH}$ through either disulfide formation or negative charge on the thiolate.

Acknowledgments: The plasmid rCDO/pET32a was a kind gift from Dr. Martha Stipanuk (Cornell University, USA). Maria Kanitz and Malcolm T. Rutledge are thanked for assistance with expression, purification and mutagenesis of CDO. This work was supported by the Marsden Fund of the Royal Society of New Zealand (GNLJ, PI; SMW, AI) and a Lottery Health (New Zealand) grant. EPT was supported by a Canadian Institutes of Health Research Postdoctoral Fellowship. ASF acknowledges the Tertiary Education Trust Fund for a studentship and SdV the National Service of Computational Chemistry Software for cpu time.

Electronic Supplementary Material Available: Coordinates used for computational calculations. 


\section{References}

1 J. E. Dominy, Jr., C. R. Simmons, L. L. Hirschberger, J. Hwang, R. M. Coloso and M. H. Stipanuk (2007) J Biol Chem 282:25189-25198

2 N. Bruland, J. H. Wubbeler and A. Steinbuchel (2009) J Biol Chem 284:660-672

3 E. P. Tchesnokov, M. Fellner, E. Siakkou, T. Kleffmann, L. W. Martin, S. Aloi, I. L. Lamont, S. M. Wilbanks and G. N. L. Jameson (2015) J Biol Chem 290:24424-24437

4 M. Fellner, S. Aloi, E. P. Tchesnokov, S. M. Wilbanks and G. N. L. Jameson (2016) Biochemistry 55:1362-1371

5 B. S. Pierce, B. P. Subedi, S. Sardar and J. K. Crowell (2015) Biochemistry 54:74777490

6 U. Brandt, M. Schurmann and A. Steinbuchel (2014) J Biol Chem 289:30800-30809

7 G. N. L. Jameson (2011) Monatsh Chem 142:325-329

8 M. Brait, S. Ling, J. K. Nagpal, X. Chang, H. L. Park, J. Lee, J. Okamura, K. Yamashita, D. Sidransky and M. S. Kim (2012) Plos One 7:e44951

9 D. Dietrich, M. Krispin, J. Dietrich, A. Fassbender, J. Lewin, N. Harbeck, M. Schmitt, S. Eppenberger-Castori, V. Vuaroqueaux, F. Spyratos, J. A. Foekens, R. Lesche and J. W. Martens (2010) BMC cancer 10:247

10 J. E. Dominy, Jr., C. R. Simmons, P. A. Karplus, A. M. Gehring and M. H. Stipanuk (2006) J Bacteriol 188:5561-5569

11 W. Li, E. J. Blaesi, M. D. Pecore, J. K. Crowell and B. S. Pierce (2013) Biochemistry 52:9104-9119

12 E. Siakkou, M. T. Rutledge, S. M. Wilbanks and G. N. L. Jameson (2011) Biochim Biophys Acta 1814:2003-2009 
13 C. G. Davies, M. Fellner, E. P. Tchesnokov, S. M. Wilbanks and G. N. L. Jameson (2014) Biochemistry 53:7961-7968

14 C. R. Simmons, Q. Liu, Q. Huang, Q. Hao, T. P. Begley, P. A. Karplus and M. H. Stipanuk (2006) J Biol Chem 281:18723-18733

15 T. Kleffmann, S. A. Jongkees, G. Fairweather, S. M. Wilbanks and G. N. Jameson (2009) J Biol Inorg Chem 14:913-921

16 S. Ye, X. Wu, L. Wei, D. Tang, P. Sun, M. Bartlam and Z. Rao (2007) J Biol Chem $282: 3391-3402$

17 C. M. Driggers, R. B. Cooley, B. Sankaran, L. L. Hirschberger, M. H. Stipanuk and P. A. Karplus (2013) J Mol Biol 425:3121-3136

18 J. E. Dominy, Jr., J. Hwang, S. Guo, L. L. Hirschberger, S. Zhang and M. H. Stipanuk (2008) J Biol Chem 283:12188-12201

19 M. Fellner, L. M. Doughty, G. N. L. Jameson and S. M. Wilbanks (2014) Anal Biochem 459:56-60

20 E. Siakkou, S. M. Wilbanks and G. N. L. Jameson (2010) Anal Biochem 405:127-131

21 R. J. Souness, T. Kleffmann, E. P. Tchesnokov, S. M. Wilbanks, G. B. Jameson and G. N. L. Jameson (2013) Biochemistry 52:7606-7617

22 J. W. Pflugrath (1999) Acta Crystallogr D Biol Crystallogr 55:1718-1725

23 A. G. W. Leslie and H. R. Powell (2007) Nato Sci Ser II Math 245:41-51

24 P. R. Evans and G. N. Murshudov (2013) Acta Crystallogr, Sect D Biol Crystallogr 69:1204-1214

25 S. Bailey (1994) Acta Crystallographica Section D, Biological Crystallography 50:760763 
26 P. D. Adams, P. V. Afonine, G. Bunkoczi, V. B. Chen, I. W. Davis, N. Echols, J. J. Headd, L. W. Hung, G. J. Kapral, R. W. Grosse-Kunstleve, A. J. McCoy, N. W. Moriarty, R. Oeffner, R. J. Read, D. C. Richardson, J. S. Richardson, T. C. Terwilliger and P. H. Zwart (2010) Acta Crystallogr D Biol Crystallogr 66:213-221

27 P. Emsley and K. Cowtan (2004) Acta Crystallogr D Biol Crystallogr 60:2126-2132

28 N. W. Moriarty, R. W. Grosse-Kunstleve and P. D. Adams (2009) Acta Crystallogr, Sect D Biol Crystallogr 65:1074-1080

29 V. B. Chen, W. B. Arendall, 3rd, J. J. Headd, D. A. Keedy, R. M. Immormino, G. J. Kapral, L. W. Murray, J. S. Richardson and D. C. Richardson (2010) Acta Crystallogr D Biol Crystallogr 66:12-21

30 M. J. Frisch, Gaussian 09 and C.01 (2010). Gaussian Inc., Wallingford, CT,

31 S. Aluri and S. P. de Visser (2007) J Am Chem Soc 129:14846-14847

32 A. D. Becke (1993) J Chem Phys 98:5648-5652

33 C. Lee, W. Yang and R. G. Parr (1988) Phys Rev B Condens Matter 37:785-789

34 P. J. Hay and W. R. Wadt (1985) J Chem Phys 82:270-283

35 E. F. Pettersen, T. D. Goddard, C. C. Huang, G. S. Couch, D. M. Greenblatt, E. C. Meng and T. E. Ferrin (2004) J Comput Chem 25:1605-1612

36 C. R. Simmons, L. L. Hirschberger, M. S. Machi and M. H. Stipanuk (2006) Protein Express Purif 47:74-81

37 M. Prudent and H. H. Girault (2009) Metallomics 1:157-165

38 J. G. McCoy, L. J. Bailey, E. Bitto, C. A. Bingman, D. J. Aceti, B. G. Fox and G. N. Phillips, Jr. (2006) Proc Natl Acad Sci U S A 103:3084-3089

39 E. J. Blaesi, B. G. Fox and T. C. Brunold (2015) Biochemistry 54:2874-2884 
40 E. I. Solomon, A. Decker and N. Lehnert (2003) Proc Natl Acad Sci U S A 100:35893594

41 L. C. Blasiak, F. H. Vaillancourt, C. T. Walsh and C. L. Drennan (2006) Nature 440:368371

42 M. L. Neidig, C. D. Brown, K. M. Light, D. G. Fujimori, E. M. Nolan, J. C. Price, E. W. Barr, J. M. Bollinger, C. Krebs, C. T. Walsh and E. I. Solomon (2007) J Am Chem Soc 129:14224-14231 
Table 1: Data collection and refinement statistics for rat CDO 164S structures. Values in parentheses indicate the highest-resolution shell.

\begin{tabular}{|c|c|c|}
\hline & C164S resting state & C164S cysteine bound \\
\hline \multicolumn{3}{|l|}{ Data collection $^{\mathrm{a}}$} \\
\hline Resolution range $(\AA)$ & $21.09-1.77(1.83-1.77)$ & $23.88-1.70(1.76-1.70)$ \\
\hline Space group & $\mathrm{P} 4_{3} 2_{1} 2$ & $\mathrm{P} 4_{3} 2_{1} 2$ \\
\hline Unit cell & 57.857 .8123 .07909090 & 58.0258 .02122 .23909090 \\
\hline Total reflections & $165,219(9,825)$ & $282,218(13,606)$ \\
\hline Unique reflections & $21,162(1,244)$ & $22,847(1,175)$ \\
\hline Multiplicity & $7.8(7.9)$ & $12.4(11.6)$ \\
\hline Anomalous multiplicity & $4.2(4.1)$ & $6.6(6.1)$ \\
\hline Completeness $(\%)$ & $99.9(100)$ & $96.7(93.1)$ \\
\hline Anomalous completeness (\%) & $100(100)$ & $97.0(92.7)$ \\
\hline Mean I/ $/ \mathrm{I}$ & $12.2(2.5)$ & $23.6(2.3)$ \\
\hline R-merge & $0.088(0.321)$ & $0.058(0.832)$ \\
\hline Anomolous R-merge & $0.084(0.301)$ & $0.056(0.799)$ \\
\hline $\begin{array}{l}\text { Half-dataset anomalous } \\
\text { difference correlation }\end{array}$ & $-0.171(-0.132)$ & $-0.042(0.037)$ \\
\hline $\mathrm{CC} 1 / 2$ & $0.996(0.946)$ & $0.999(0.843)$ \\
\hline \multicolumn{3}{|l|}{ Refinement $^{\text {b }}$} \\
\hline Reflections for refinement & 21,073 & 22,818 \\
\hline R-work & $0.175(0.229)$ & $0.178(0.244)$ \\
\hline R-free & $0.208(0.301)$ & $0.206(0.271)$ \\
\hline RMS bonds $(\AA)$ & 0.008 & 0.009 \\
\hline RMS angles $\left(^{\circ}\right)$ & 1.08 & 1.10 \\
\hline Ramachandran favored (\%) & 98.4 & 99.5 \\
\hline Ramachandran outliers (\%) & 0 & 0 \\
\hline Rotamer outliers $(\%)$ & 0.6 & 0.6 \\
\hline Clashscore & 5.47 & 3.28 \\
\hline Average B-factor $\left(\AA^{2}\right)$ & 24.1 & 29.9 \\
\hline Macromolecules $\left(\AA^{2}\right)$ & 22.1 & 28.5 \\
\hline Iron atom $\left(\AA^{2}\right)$ & 14.7 & 21.1 \\
\hline Cysteine $\left(\AA^{2}\right)$ & - & 25.1 \\
\hline Solvent $\left(\AA^{2}\right)$ & 34.1 & 38.9 \\
\hline PDB ID & $4 \mathrm{YYO}$ & $4 Z 82$ \\
\hline
\end{tabular}

${ }^{\mathrm{a}}$ Data collection statistics as reported by aimless.[24]

${ }^{\mathrm{b}}$ Refinement statistics as reported by phenix.refine [26] and Molprobity. [29] 


\section{Figure Legends}

Figure 1. Plot of $k_{\mathrm{obs}}$ of cysteine dioxygenase activity of WT (full circles) and C164S (open circles) as a function of cysteine concentration at $\mathrm{pH} 5.7,6.5$ and 7.5. The data can be fitted to the Michaelis-Menten equation (solid lines) with parameters: $\mathrm{pH} 5.7\left(k_{\mathrm{cat}}=0.04 \pm 0.07 \mathrm{~s}^{-1}\right.$ and $\left.K_{\mathrm{m}}=3 \pm 2 \mathrm{mM}\right), \mathrm{pH} 6.5\left(k_{\mathrm{cat}}=0.40 \pm 0.07 \mathrm{~s}^{-1}\right.$ and $\left.K_{\mathrm{m}}=11 \pm 2 \mathrm{mM}\right), \mathrm{pH} 7.5\left(\mathrm{C} 164 \mathrm{~S} k_{\mathrm{cat}}=0.48 \pm\right.$ $0.07 \mathrm{~s}^{-1}$ and $K_{\mathrm{m}}=5 \pm 2 \mathrm{mM}$; WT $k_{\mathrm{cat}}=0.40 \pm 0.07 \mathrm{~s}^{-1}$ and $\left.K_{\mathrm{m}}=9 \pm 2 \mathrm{mM}\right)$.

Figure 2. (A) Reaction profile of $\mathrm{C} 164 \mathrm{~S}(2.2 \mu \mathrm{M})$ at $\mathrm{pH} 7.5,37^{\circ} \mathrm{C}$ showing cysteine depletion (full squares, left axis), CSA formation (full circles) and \% crosslink (open circles, right axis) as a function of time. (B) Final \% crosslink formed after reaction as a function of $\mathrm{pH}$. WT (full triangles) and C164S (open circles) show greater differences at higher $\mathrm{pH}$.

Figure 3. Crystal structure of the resting state and cysteine bound C164S CDO variant. Figures and alignments created by UCSF Chimera [35] using coloring-by-element scheme except carbon atoms, iron atoms and iron coordination in black (resting state C164S, PDB entry 4YYO), in cyan (resting state WT, 4KWJ [21]), in purple (cysteine bound C164S, 4Z82) and silver (cysteine bound WT, 4IEV [17]). (A) Active site of the resting state C164S CDO variant. $2 m F_{o}-D F_{c}$ active site electron density (at 1 RMSD, blue), the anomalous map (at 3 RMSD, yellow) and the refined model. (B) Structural comparison of the resting states of C164S and WT CDO using a C $\alpha$ atom alignment. (C) Unbiased active site density of the cysteine bound C164S CDO variant before model building. Final model compared to a $m F_{o}-D F_{c}$ electron density map (green) based on a model with no iron, cysteine or water atoms and no sidechains for arginine 60 , cysteine 93 and tyrosine 157 and anomalous map (at 3 RMSD, yellow). (D) Active site of the 
cysteine bound C164S CDO variant. $2 m F_{o}-D F_{c}$ active site electron density (at 1 RMSD, blue), the anomalous map (at 3 RMSD, yellow) and the refined model. (E) Structural comparison of the cysteine bound $\mathrm{C} 164 \mathrm{~S}$ and WT CDO structure using a $\mathrm{C} \alpha$ atom alignment.

Figure 4. Comparison of hydrogen bonding in A) WT (PDB 4IEV) and B) C164S variant of CDO (PDB 4Z82). Figures created and colored as in Figure 3. Proposed hydrogen bonds are shown in red.

Figure 5. B3LYP optimized geometry of the resting state complex (above) and C164S (below) as obtained in Gaussian-09. Bond lengths are in angstroms and the charge $(Q)$ is in au. Details at right include iron, substrate cysteine, residue 164 and associated water molecule, rotated relative to main figures by approximately $90^{\circ}$ around a horizontal axis in the plane of the page. 
Figures
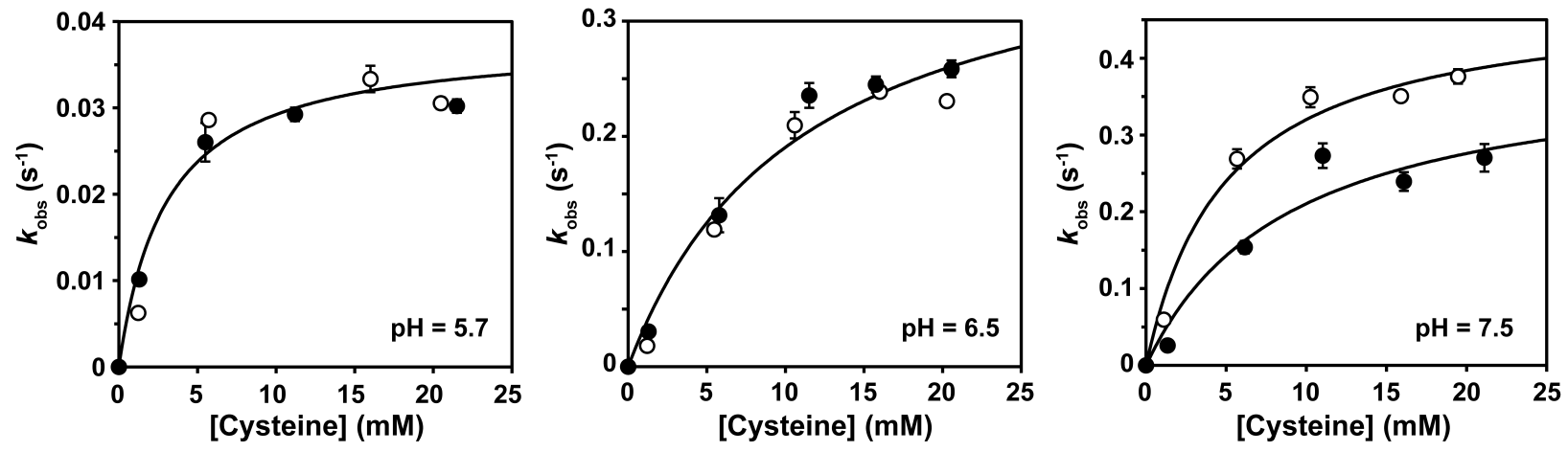

Figure 1. 

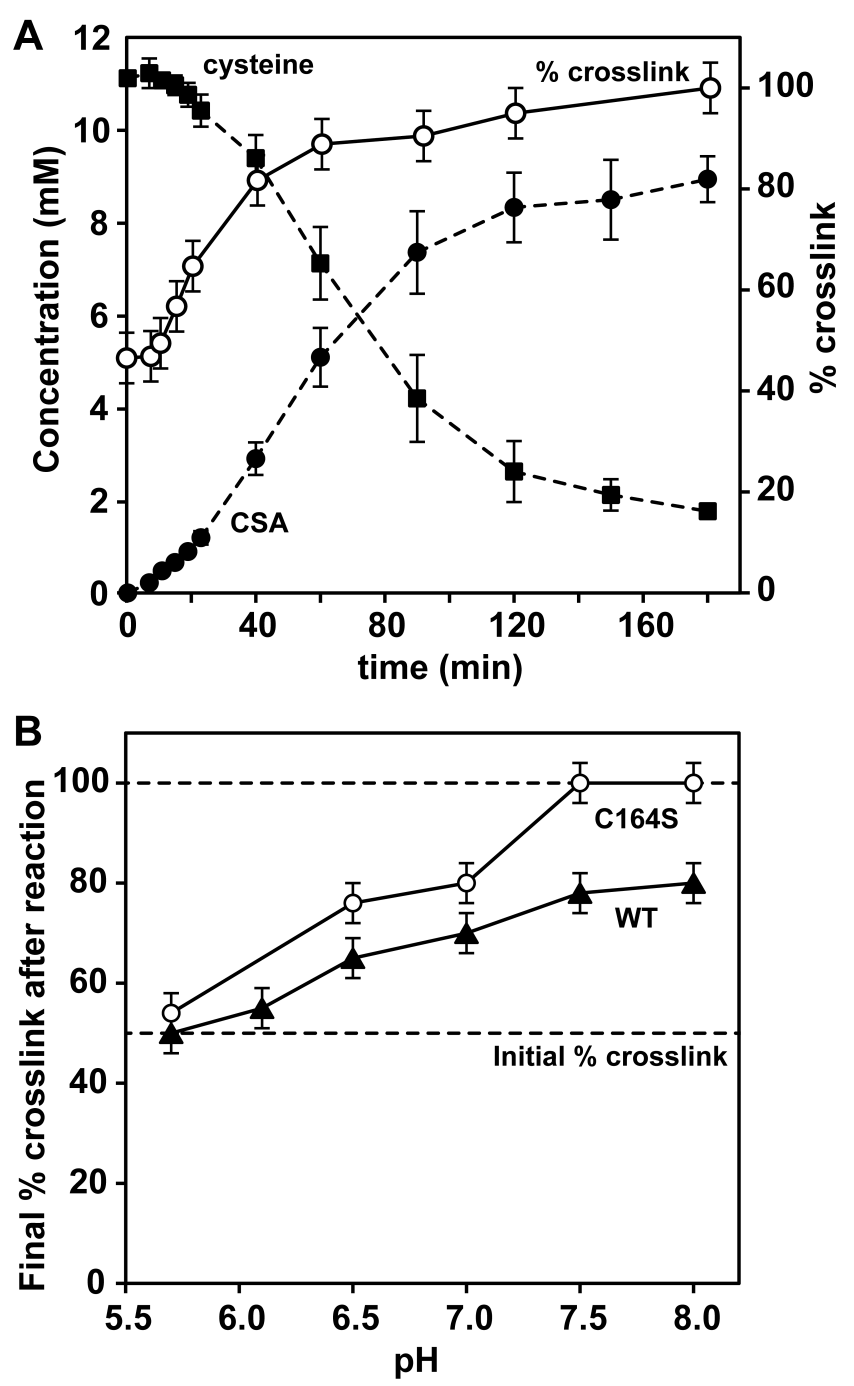

Figure 2. 

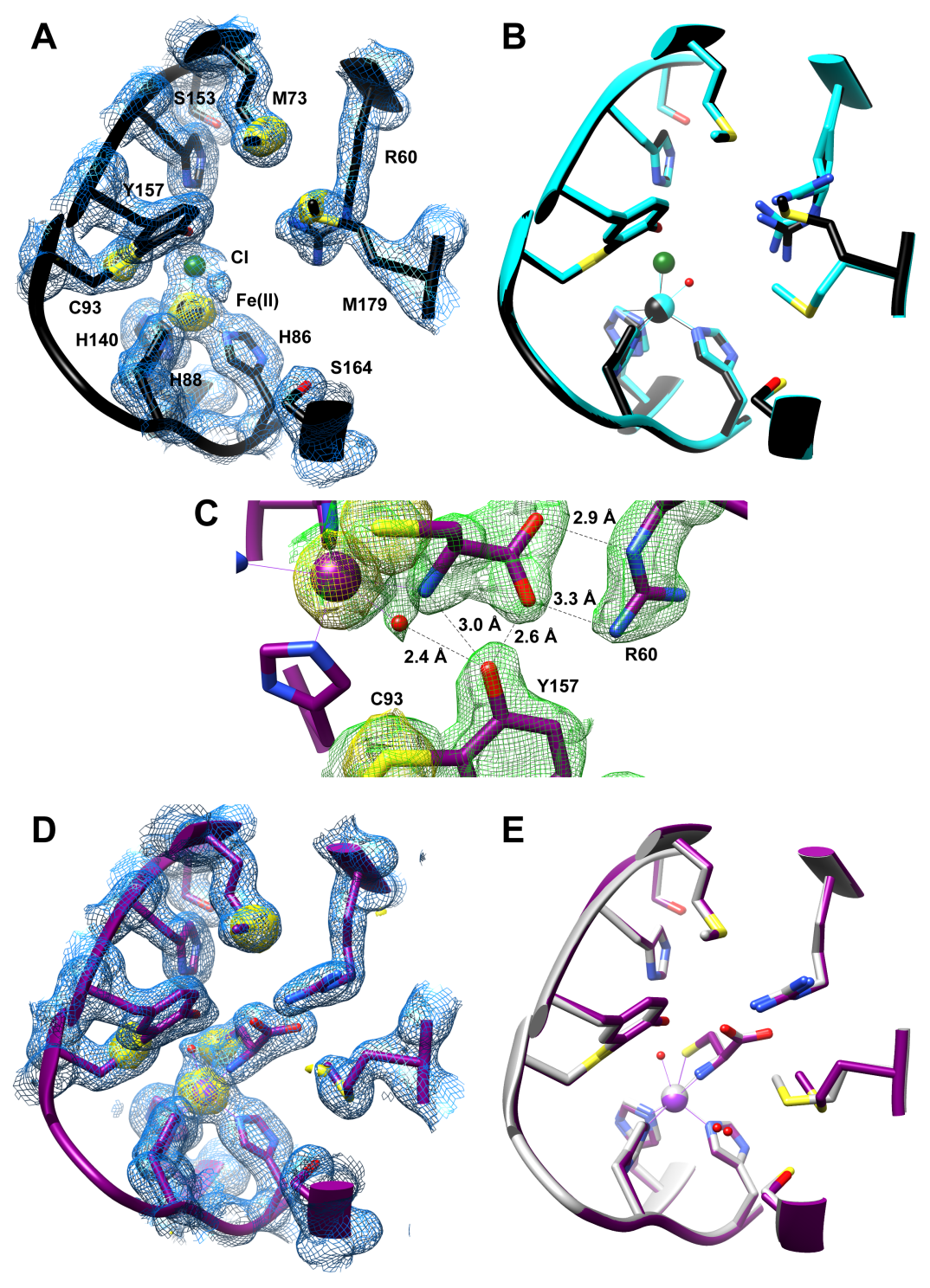

Figure 3. 
A

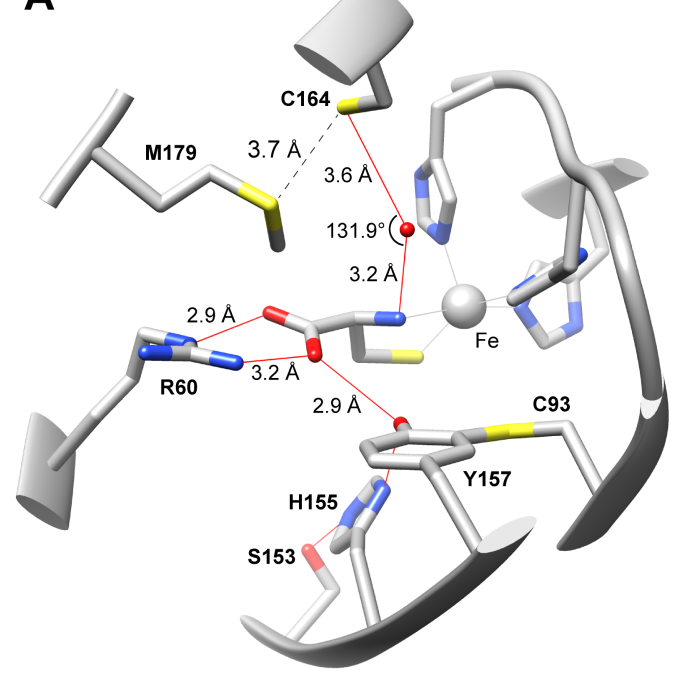

B

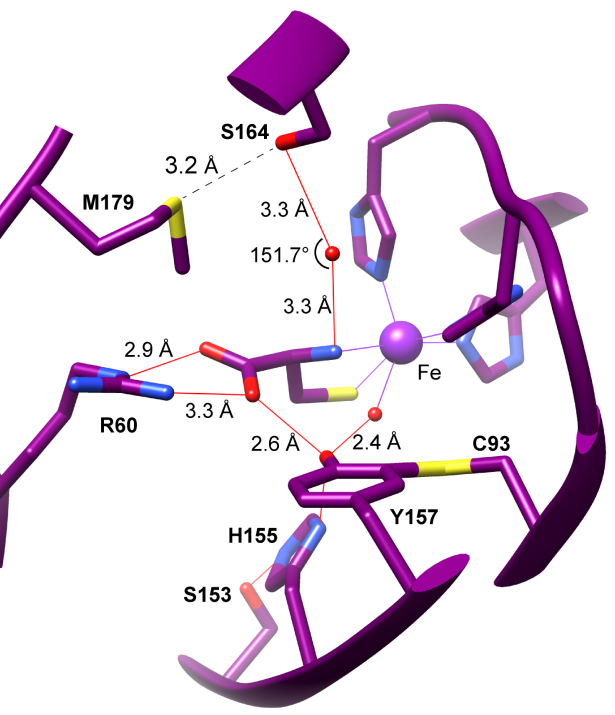

Figure 4. 


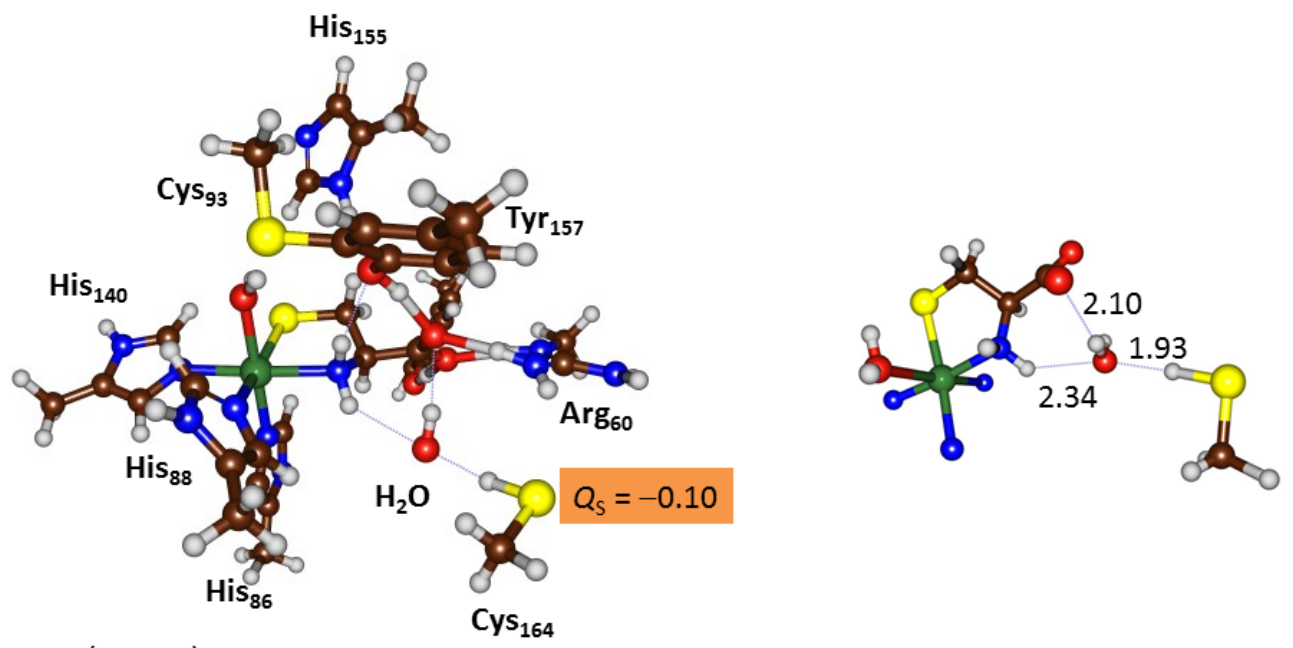

$\mathrm{Fe}-\mathrm{N}($ His140) $=2.21$

$\mathrm{Fe}-\mathrm{N}(\mathrm{His} 88)=2.21$

$\mathrm{Fe}-\mathrm{N}(\mathrm{His} 86)=2.21$

$\mathrm{Fe}-\mathrm{OH}_{2}=2.19$

Fe-SCys $=2.57$

Fe-NCys $=2.28$

WT CDO model viewed from two angles.
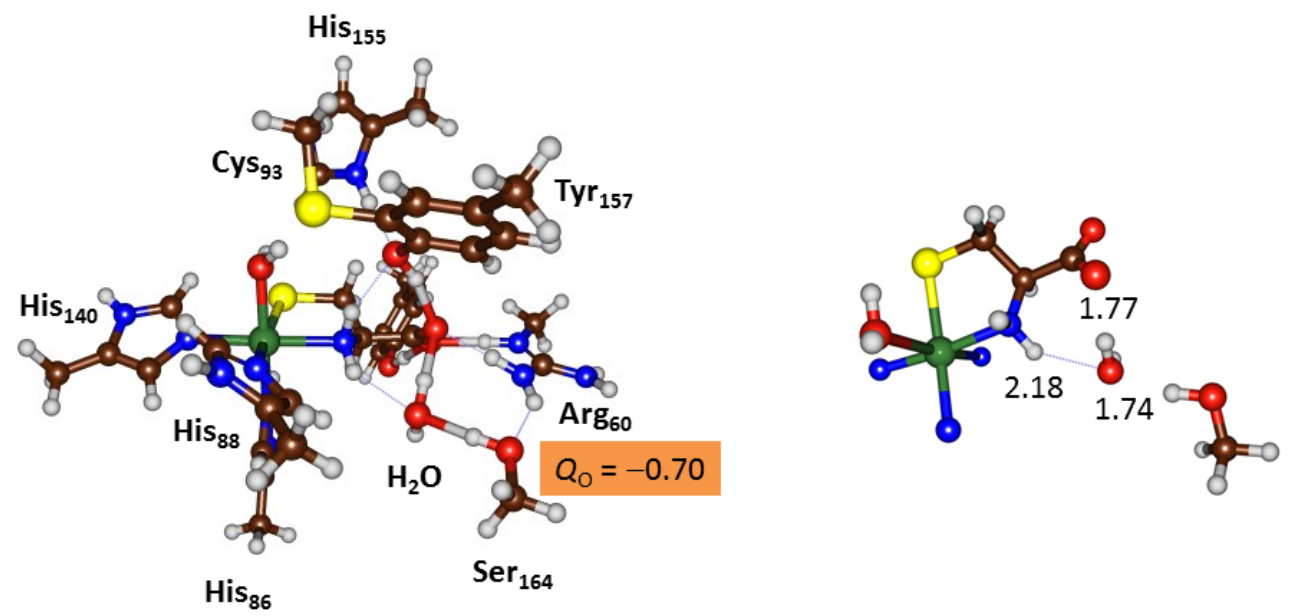

$\mathrm{Fe}-\mathrm{N}(\mathrm{His} 140)=2.21$

$\mathrm{Fe}-\mathrm{N}($ His88) $=2.21$

$\mathrm{Fe}-\mathrm{N}(\mathrm{His} 86)=2.21$

$\mathrm{Fe}-\mathrm{OH}_{2}=2.17$

$\mathrm{Fe}-\mathrm{SCys}=2.61$

Fe-NCys $=2.26$

C164S CDO model viewed from two angles.

Figure 5. 